\title{
膨润土负载红磷复合材料的吸附富集-定位光降解性能
}

\author{
朱恩权，马玉花，艾尼瓦・木尼热，粟 智 \\ (新疆师范大学 化学化工学院, 乌鲁木齐 830054)
}

摘 要: 为了提高红磷催化剂的光催化性能, 选择剥离膨润土(EB)为载体, 将水热处理后的红磷(HRP)负载在 $\mathrm{EB}$ 上, 制得 $\mathrm{EB} / \mathrm{HRP}$ 复合光催化剂, 并通过不同手段对催化剂进行表征。选择罗丹明 $\mathrm{B}$ 为模型污染物, 考察了 $\mathrm{EB} / \mathrm{HRP}$ 复 合光催化剂的光降解性能。结果表明, 随着 $\mathrm{EB}$ 含量的增加, $\mathrm{EB} / \mathrm{HRP}$ 复合光催化剂的光降解效率呈现先增加后减小 的趋势, 当 $\mathrm{EB}$ 的质量分数为 $9 \%$ 时, 复合光催化剂 $\left(\mathrm{EB}_{9} / \mathrm{HRP}\right)$ 表现出最强的吸附性能和光降解性能, 其降解速率常 数 $k$ 值为 $0.0641 \mathrm{~min}^{-1}$, 是 HRP 的 2 倍。另外, 经过五次循环光降解实验, $\mathrm{EB}_{9} / \mathrm{HRP}$ 仍具有较高的光催化活性 $(96.8 \%)$ 。 因此, $\mathrm{EB}_{9} / \mathrm{HRP}$ 复合催化剂具有较好的光催化活性和稳定性, 有望成为一种降解污染物的高效而稳定的光催化剂。 关 键 词: 红磷; 膨润土; 吸附富集-定位光降解; 光催化剂

中图分类号: O643 文献标识码: A

\section{Adsorption-enrichment and Localized-photodegradation of Bentonite-supported Red Phosphorus Composites}

\author{
ZHU Enquan, MA Yuhua, AINIWA • Munire, SU Zhi \\ (College of Chemistry and Chemical Engineering, Xinjiang Normal University, Urumqi 830054, China)
}

\begin{abstract}
The hydrothermally treated red phosphorus (HRP) was dispersed on exfoliated bentonite (EB) supporter to prepare the EB/HRP photocatalyst for improving photocatalytic performance. The as-synthesized samples were characterized by different methods. Rhodamine B was selected as the model pollutant to evaluate the photodegradation property of EB/HRP. Results showed that the photodegradation efficiency of the EB/HRP photocatalyst composite increased with increased EB mass fraction, and decreased after reaching the highest value. When the mass fraction of $\mathrm{EB}$ was $9 \%$, the $\mathrm{EB}_{9} / \mathrm{HRP}$ photocatalyst composite exhibited the maximum adsorption performance and photodegradation activity. Its degradation rate constant $k$ was $0.0641 \mathrm{~min}^{-1}$, which was two times that of HRP. In addition, after five cycles of photodegradation experiments, $\mathrm{EB}_{9} / \mathrm{HRP}$ still had high photocatalytic activity (96.8\%). Therefore, the $\mathrm{EB}_{9} / \mathrm{HRP}$ catalyst composite had good photocatalytic activity and stability, which can be an efficient
\end{abstract}

收稿日期：2019-08-12；收到修改稿日期：2019-11-07

基金项目: 新疆维吾尔自治区自然科学基金(2019D01B36，2019D01A69); 自治区“百名青年博士引进计划”天池博士项目 (BS2017002); 自治区高校科研计划项目(XJEDU2018Y030); 新疆维吾尔自治区“天山青年计划”优秀青年科技 人才项目 (2017Q027); 新疆师范大学博士科研启动项目(XJNUBS1907); 新疆师范大学“十三五”校级重点学科 招标课题(17SDK0802); 国家自然科学基金(21862022)

Natural Science Foundation of Xinjiang Uygur Autonomous Region (2019D01B36, 2019D01A69); Dr. Tianchi of the "Hundred Young Doctors Introduction Program" of the Autonomous Region (BS2017002); Natural Science Youth Project in Universities and Colleges of the Autonomous Region (XJEDU2018Y030); Tianshan Youth Talents Plan Project of Xinjiang (2017Q027), Ph.D. Startup Fund of Xinjiang Normal University (XJNUB1907); "13th Five-Year" Plan for Key Discipline Bidding Project of Xinjiang Normal University (17SDK0802); National Natural Science Foundation of China (21862022)

作者简介: 朱恩权(1992-), 男, 硕士研究生. E-mail: 1427830223@qq.com ZHU Enquan (1992-), male, Master candidate. E-mail: 1427830223@qq.com

通讯作者：马玉花，讲师.E-mail: 15199141253@163.com; 粟 智，教授.E-mail: suzhixj@sina.com MA Yuhua, lecturer. E-mail: 15199141253@163.com; SU Zhi, professor. E-mail: suzhixj@sina.com 
and stable photocatalyst for the degradation of pollutants.

Key words: red phosphorus; bentonite; adsorption enrichment-localized photodegradation; photocatalyst

半导体辅助光降解有机污染物是一种经济、环 保的环境污染治理方案, 受到了广泛关注。近年来, 研究发现硒、硫、红磷(RP) 等单质半导体材料的带 隙窄、可见光响应强, 在光催化领域有着极大的应 用潜力。其中, RP 在可见光照射下不仅可以分解水, 还能降解污染物, 并且在地壳中储存丰富, 因此是 一种很有前景的元素半导体光催化剂 ${ }^{[1-4]}$ 。 RP 的禁 带宽度约为 $1.7 \mathrm{eV}$, 吸收带边位置达到 $680 \mathrm{~nm}$, 是 一种典型的对可见光响应的 $\mathrm{P}$ 型半导体材料 ${ }^{[1]}$, 被 列为收集太阳能用于环境净化和制备清洁能源最有 前景的光催化剂之一。此外, 通过控制 RP 晶体的形 貌 $^{[5-7]}$ 和晶面 ${ }^{[8]}$, 或者与其它材料复合, 可以进一步 提高 RP 的光催化活性。其中, 已报道的与 RP 成功 复合的材料有 $\mathrm{CdS}^{[9]} 、 \mathrm{MoS}_{2}{ }^{[10-11]} 、 \mathrm{YPO}_{4}{ }^{[2]} 、 \mathrm{TiO}_{2}{ }^{[12]}$ 、 $\mathrm{C}_{3} \mathrm{~N}_{4}{ }^{[3,13-14]}$ 、黑磷 ${ }^{[4]}$ 以及石墨烯 ${ }^{[15]}$ 、还原氧化石墨烯 ${ }^{[11]}$ 等。然而, 上述材料或较难制备或制备成本高, 复合 催化剂难以进行大规模生产。因此, 寻找一种低成 本、易获得, 并能进一步增强 RP 光催化性能的材料 具有十分重要的意义。

膨润土具有成本低、易获得、环境友好等优点, 是各种光催化剂的理想负载材料。膨润土是蒙脱石 的工业名称, 是一种黏土岩, 由 $2: 1$ 的堆叠层组成, 两层四面体片夹着八面体片, 具有膨胀现象 ${ }^{[16]}$ 。在 硅氧四面体中 $\mathrm{Al}^{3+}$ 对 $\mathrm{Si}^{4+}$ 的同晶取代和铝氧八面体 中 $\mathrm{Al}^{3+}$ 对 $\mathrm{Mg}^{2+}$ 或 $\mathrm{Zn}^{2+}$ 的同晶取代导致网状黏土表面 产生负电荷 ${ }^{[17]}$ 。与其它黏土相比, 膨润土具有优异 的吸附能力, 在其层间、外部表面和边缘具有丰富 的吸附/交换活性位点 ${ }^{[18]}$ 。因此, 膨润土是去除染料 的最有效吸附剂 ${ }^{[19]}$ 。许多研究还表明, 膨润土是合 成具有较高光催化活性的复合光催化剂的良好载体, 例如 $\mathrm{TiO}_{2} /$ 膨润土 ${ }^{[20-21]} 、 \mathrm{ZnO} /$ 膨润土 ${ }^{[22]} 、 \mathrm{Bi}_{2} \mathrm{O}_{3} /$ 膨润 土 ${ }^{[23]} 、 \mathrm{~g}-\mathrm{C}_{3} \mathrm{~N}_{4} / \mathrm{Ag}_{3} \mathrm{PO}_{4} /$ 膨润土 ${ }^{[24]} 、 \mathrm{BiVO}_{4} /$ 膨润土 ${ }^{[25]}$ 和 $\mathrm{Ni}_{2} \mathrm{O}_{3} /$ 膨润土 ${ }^{[26]}$ 等。由于光催化剂在膨润土基体 上均匀分散, 产生了明显的反应活性位点, 这些复 合光催化剂对有机污染物表现出很强的降解能力。 此外, 所有膨润土合成的复合材料都具有更大的比 表面积和更高的吸附性能, 使得复合催化剂比纯相 具有更高的光催化性能, 并且催化剂在黏土上充分 分散, 减少了降解有机污染物所需的光催化剂质量。

本工作将水热处理后的 RP(HRP)组装到剥离膨 润土纳米膜上, 制备了剥离膨润土/红磷(EB/HRP) 纳米复合材料, 并将其应用于光降解有机染料。对
合成的 $\mathrm{EB} / \mathrm{HRP}$ 复合材料的成分、电化学性质等进 行了表征。通过对罗丹明 $\mathrm{B}(\mathrm{RhB})$ 进行吸附和光降解 实验, 评价 $\mathrm{EB} / \mathrm{HRP}$ 复合材料的吸附富集一定位光降 解性能以及剥离膨润土含量对复合材料的影响, 并提 出了 $\mathrm{EB} / \mathrm{HRP}$ 复合材料光降解有机污染物的可能机理。

\section{1 实验方法}

\section{1 膨润土负载红磷复合光催化剂的制备}

水热法处理 RP: 准确称取 $0.6 \mathrm{~g}$ 的商业红磷 (CRP)分散于 $20 \mathrm{~mL}$ 蒸馏水中后置于反应釜中, 在 $200{ }^{\circ} \mathrm{C}$ 恒温箱中水热处理 $12 \mathrm{~h}$, 结束后离心、洗涤、 干燥 $\left(80{ }^{\circ} \mathrm{C}, 4 \mathrm{~h}\right)$, 即可获得水热处理后的 RP, 标记 为 HRP。

剥离膨润土的制备: 称取 $4 \mathrm{~g}$ 膨润土分散于 $70 \mathrm{~mL}$ 去离子水中, 磁力搅拌 $1.5 \mathrm{~h}$, 静置过夜。将 下层大颗粒物质去掉, 上层凝胶状物质离心, 去离 子水洗涤数次后在 $80{ }^{\circ} \mathrm{C}$ 干燥。加入一定量 $0.5 \mathrm{~mol} / \mathrm{L}$ 稀硝酸, 搅拌过夜, 离心除去上层液体, 并用去离 子水洗涤数次, 在 $80{ }^{\circ} \mathrm{C}$ 的恒温箱中干燥过夜, 获 得剥离膨润土备用, 并标记为 $\mathrm{EB}$ 。

EB/HRP 复合光催化剂的制备: 按照 $\mathrm{EB}$ 质量 分数为 $(3 \%, 5 \%, 7 \%, 9 \%, 11 \%)$ 与 $\mathrm{HRP}$ 进行充分混合, 随后将混合溶液置于 $50 \mathrm{~mL}$ 反应釜于 $150{ }^{\circ} \mathrm{C}$ 下水热 处理 $4 \mathrm{~h}$, 将所得沉淀物离心、洗涤、干燥 $\left(80{ }^{\circ} \mathrm{C}, 4 \mathrm{~h}\right)$ 得到催化剂, 分别标记为 $\mathrm{EB}_{3} / \mathrm{HRP}, \mathrm{EB}_{5} / \mathrm{HRP}, \mathrm{EB}_{7} / \mathrm{HRP}$, $\mathrm{EB}_{9} / \mathrm{HRP}, \mathrm{EB}_{11} / \mathrm{HRP}$ 。

\section{$1.2 \mathrm{~EB} / \mathrm{HRP}$ 复合光催化剂的性能表征}

采用 X 射线衍射仪(XRD, D8 Advance)分析催 化剂的物相组成; 采用傅里叶变换红外光谱仪 (FT-IR，TENSOR27)分析样品中的官能团; 采用场 发射扫描电子显微镜(FESEM, JSM-7610F)表征样 品的形貌; 采用比表面分析仪(BET, Autosorb-IQ2MP)测试样品的比表面积和孔径; 利用电化学工作 站(CHI660E)测试催化剂的电化学性质, 工作电极 为制备的催化剂, 对电极为 $\mathrm{Pt}$ 电极, 参比电极为 $\mathrm{Ag} / \mathrm{AgCl}$ 电极, 电解液为 $0.1 \mathrm{~mol} / \mathrm{L} \mathrm{Na}_{2} \mathrm{SO}_{4}$ 溶液。

\section{$1.3 \mathrm{~EB} / \mathrm{HRP}$ 复合催化剂的光催化性能测试}

以 $\mathrm{RhB}$ 和对硝基苯酚(4-NP)为目标污染物，以 $300 \mathrm{~W}$ 氙灯光源为模拟光源, 考察 EB/HRP 的光降 解能力。具体过程如下: 取 $5 \mathrm{mg}$ 的 $\mathrm{EB} / \mathrm{HRP}$ 复合光 催化剂放入 $10 \mathrm{mg} / \mathrm{L} 、 20 \mathrm{~mL} \mathrm{RhB}$ 溶液中, 暗环境下 
磁力搅拌 $30 \mathrm{~min}$, 达到吸附-脱附平衡; 然后在光照 条件下继续搅拌, 每间隔 $10 \mathrm{~min}$ 取 $3 \mathrm{~mL}$ 上清液进 行过滤 $(0.45 \mu \mathrm{m}$ 滤膜 $)$ 。上清液置于比色血中, 用紫 外-可见分光光度计在 $\mathrm{RhB}$ 和 4-NP 最大吸收波长 554 和 $318 \mathrm{~nm}$ 处测定其吸光度。

根据公式(1)计算降解率:

$$
\text { 降解率 }=\left(C_{0}-C_{t}\right) / C_{0} \times 100 \%
$$

式中, $C_{0}$ 为 $\mathrm{RhB}$ (或 4-NP) 的原始浓度, $C_{t}$ 为光照不同 时间 $\mathrm{RhB}$ (或 4-NP)剩余的浓度。

\section{2 结果与讨论}

\section{$2.1 X R D$ 及红外光谱分析}

对 EB、HRP 和 EB-HRP 复合材料进行 XRD 分 析, 结果如图 1(a)所示。其中, EB 出现很多膨润土 的特征衍射峰, 典型的特征峰出现在 $2 \theta=27.1^{\circ}$ 位置 处。HRP 在 $2 \theta=15.6^{\circ}$ 处的峰与非晶态红磷(102) 晶面 对应, 与文献报道一致 ${ }^{[1]}$ 。从图 1 可以看出, 复合催 化剂 $\mathrm{EB}_{9} / \mathrm{HRP}$ 的 XRD 图谱上同时出现了上述红磷 和膨润土的特征衍射峰, 证明实验已经将 RP 负载 在膨润土上。

图 1(b)给出了 $\mathrm{EB} 、 \mathrm{HRP}$ 和 $\mathrm{EB}_{9} / \mathrm{HRP}$ 的 FT-IR 图谱。对于 $\mathrm{EB}, 3435 \mathrm{~cm}^{-1}$ 左右的宽带吸收对应羟基中 $\mathrm{O}-\mathrm{H}$ 键的拉伸振动; $1640 \mathrm{~cm}^{-1}$ 处的吸收峰对应吸附
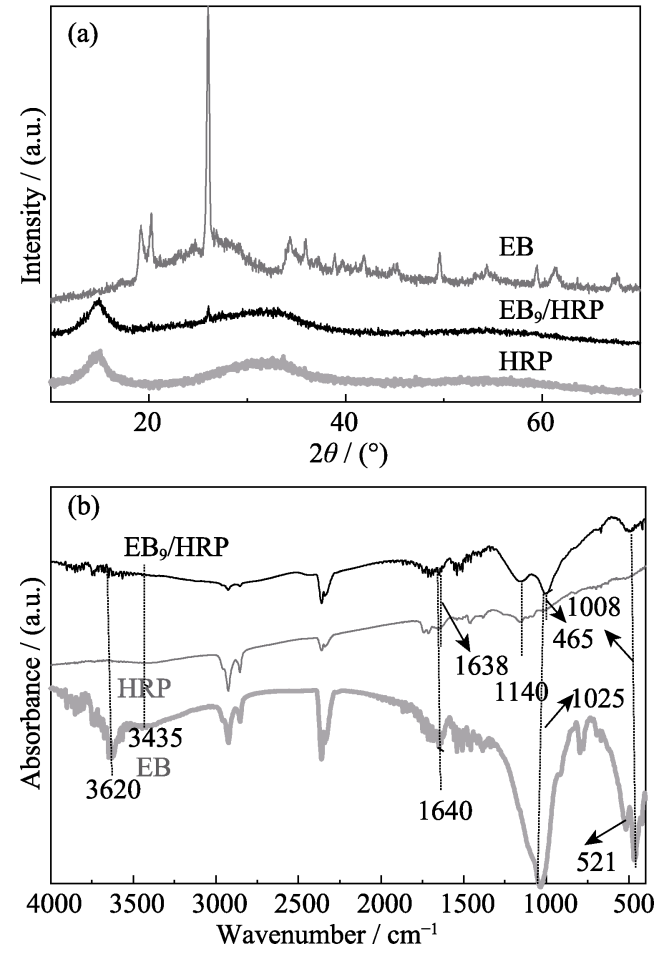

图 $1 \mathrm{~EB} 、 \mathrm{HRP}$ 和 $\mathrm{EB}_{9} / \mathrm{HRP}$ 的 XRD 图谱(a)和 FT-IR 图谱(b) Fig. 1 XRD patterns (a) and FT-IR spectra (b) of EB, HRP and $\mathrm{EB}_{9} / \mathrm{HRP}$
$\mathrm{H}_{2} \mathrm{O}$ 的 $\mathrm{H}-\mathrm{O}-\mathrm{H}$ 键的弯曲振动; $3620 \mathrm{~cm}^{-1}$ 处的吸收 峰对应 $\mathrm{Al}-\mathrm{O}-\mathrm{H}$ 拉伸振动; $1025 \mathrm{~cm}^{-1}$ 处的强吸收与 $\mathrm{Si}-\mathrm{O}-\mathrm{Si}$ 键的拉伸振动有关, 这是蒙脱石的特征吸 收峰; 465 和 $521 \mathrm{~cm}^{-1}$ 处的吸收峰对应 $\mathrm{Si}-\mathrm{O}-\mathrm{Si}$ 键和 $\mathrm{Al}-\mathrm{O}-\mathrm{Si}$ 键的弯曲振动 ${ }^{[27]}$ 。而对于纯 $\mathrm{HRP}, 1008 \mathrm{~cm}^{-1}$ 处的吸收峰对应 $\mathrm{P}-\mathrm{P}-\mathrm{O}$ 键震动吸收; $1140 \mathrm{~cm}^{-1}$ 处出 现了 $\mathrm{P}-\mathrm{O}$ 键吸收峰; $1638 \mathrm{~cm}^{-1}$ 处的弱峰对应于 $\mathrm{P}=\mathrm{O}$ 键，与文献报道 RP 特征吸收峰一致 ${ }^{[28]}$ 。 $\mathrm{EB}$ 和 HRP 的特征吸收峰在 $\mathrm{EB}_{9} / \mathrm{HRP}$ 的红外谱图中均能观察 到, 说明 $\mathrm{EB}$ 与 $\mathrm{HRP}$ 已充分复合, 这与 XRD 分析结 果一致。

\subsection{SEM 及 BET 分析}

图 2 是 $\operatorname{HRP}(\mathrm{a}, \mathrm{b})$ 和 $\mathrm{EB}_{9} / \mathrm{HRP}(\mathrm{c}, \mathrm{d})$ 的 SEM 照片, 从图中可以看出, $\mathrm{CRP}$ 经 $200{ }^{\circ} \mathrm{C}$ 水热处理后, 颗粒 粒径较小, 表面有蜂窝状的空隙, 有利于与染料充 分接触。与 $\mathrm{EB}$ 土复合后可以看到 $\mathrm{HRP}$ 附着在 $\mathrm{EB}$ 表面，形成三维的立体结构，与未复合前的紧密结 构相比，比表面积增加了，同时暴露出更多的反应 活性位点, 这将大大增强其催化活性。催化剂的 BET 分析结果表明, HRP 的比表面积为 $12.9 \mathrm{~m}^{2} / \mathrm{g}$, 孔径为 $37.0 \mathrm{~nm}$; 负载 $\mathrm{EB}$ 的催化剂 $\mathrm{EB}_{9} / \mathrm{HRP}$ 的比表 面积为 $13.9 \mathrm{~m}^{2} / \mathrm{g}$, 孔径为 $44.2 \mathrm{~nm}$ 。结果显示, HRP 固载于 $\mathrm{EB}$ 后, 其孔径和比表面积均增大,有利于污 染物的吸附富集。

\section{3 光催化性能分析}

图 3(a)反映了 $H R P$ 和 $E B / H R P$ 光催化剂对 $R h B$ 的光降解情况，黑暗条件下磁力搅拌 $30 \mathrm{~min}$ 左右可 以达到吸附-脱附平衡, 约 $40 \%$ 的 $\mathrm{RhB}$ 被 $\mathrm{EB}_{9} / \mathrm{HRP}$ 吸附，而纯 HRP 对 RhB 的吸附量仅为 $20 \%$, 表明剥 离膨润土具有较高的吸附能力, 能富集污染物。随 着光照时间的延长, $\mathrm{RhB}$ 的浓度逐渐降低, 该结果 表明，所制备的 HRP 和所有 EB/HRP 复合材料均表
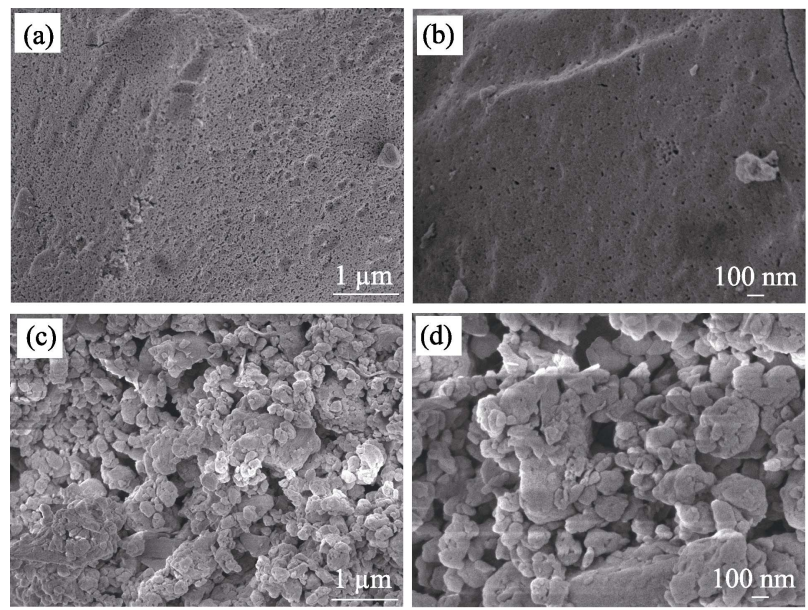

图 $2 \operatorname{HRP}(\mathrm{a}, \mathrm{b})$ 和 $\mathrm{EB}_{9} / \mathrm{HRP}(\mathrm{c}, \mathrm{d})$ 的 $\operatorname{SEM}$ 照片

Fig. 2 SEM images of (a, b) HRP and (c, d) EB $/$ HRP 

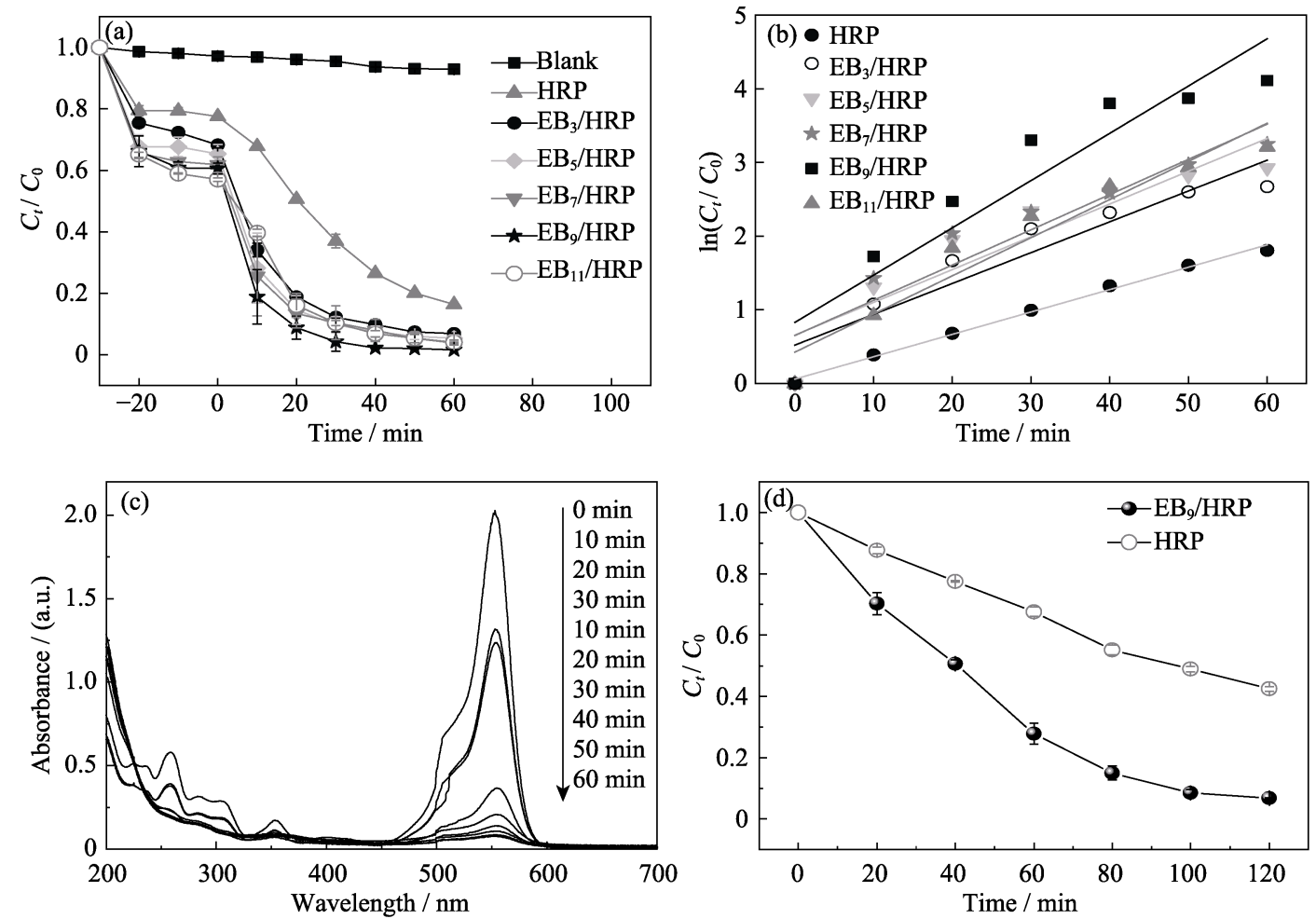

图 $3 \mathrm{HRP}$ 和 $\mathrm{EB} / \mathrm{HRP}$ 光催化剂对 $\mathrm{RhB}$ 的降解曲线(a)和反应动力学曲线(b); RhB 在催化剂 $\mathrm{EB}_{9} / \mathrm{HRP}$ 作用下的紫外-可见光谱变化图(c); HRP 和 $\mathrm{EB}_{9} / \mathrm{HRP}$ 光催化剂对 4-NP 的降解曲线(d)

Fig. 3 (a) Photocatalytic degradation of RhB by HRP and EB/HRP; (b) Reaction kinetics of RhB by HRP and EB/HRP; (c) UV-visible spectral changes of RhB over the $\mathrm{EB}_{9} / \mathrm{HRP}$; (d) Photocatalytic degradation of p-nitrophenol by $\mathrm{HRP}$ and $\mathrm{EB}_{9} / \mathrm{HRP}$

现出可见光响应的催化活性。HRP 光降解 RhB $60 \mathrm{~min}$, 其降解率仅达到 $80 \%$ 左右, 而 $\mathrm{EB} / \mathrm{HRP}$ 对 $\mathrm{RhB}$ 降解 $60 \mathrm{~min}$ 后, 其降解率均超过 $90 \%$, 降解率由大到小 排序为: $\mathrm{EB}_{9} / \mathrm{HRP}>\mathrm{EB}_{11} / \mathrm{HRP}>\mathrm{EB}_{7} / \mathrm{HRP}>\mathrm{EB}_{5} / \mathrm{HRP}>$ $\mathrm{EB}_{3} / \mathrm{HRP}$ 。EB/HRP 复合材料光催化活性的增强可 归功于催化剂的高吸附活性和大的光生载流子迁移 率。实验证明, $\mathrm{EB}_{9} / \mathrm{HRP}$ 的催化性能最好, 即 $\mathrm{EB}$ 的 最佳负载量确定为 $9 \%$ 。

图 3(b)为 $\mathrm{HRP}$ 和 $\mathrm{EB} / \mathrm{HRP}$ 光降解 $\mathrm{RhB}$ 的动力 学曲线, 符合一级动力学模型, 公式如下:

$$
\ln \left(C_{t} / C_{0}\right)=-k t
$$

其中, $k$ 为速率常数。由图可以得到 $\mathrm{EB}_{3} / \mathrm{HRP}$ 、

$\mathrm{EB}_{5} / \mathrm{HRP} 、 \mathrm{~EB}_{7} / \mathrm{HRP} 、 \mathrm{~EB}_{9} / \mathrm{HRP}$ 和 $\mathrm{EB}_{11} / \mathrm{HRP}$ 的速率常 数分别为 $0.0418 、 0.0444 、 0.0478 、 0.0641$ 和 $0.0517 \mathrm{~min}^{-1}$, 均大于 HRP 的速率常数 $\left(0.0303 \mathrm{~min}^{-1}\right)$ 。该结果表明 $\mathrm{EB} / \mathrm{HRP}$ 复合材料在可见光区域对 RhB 降解能力比 $\mathrm{HRP}$ 的强, 其中 $\mathrm{EB}_{9} / \mathrm{HRP}$ 展现出最高的光催化活性。

结合图 3(a,b) 可知, 与 $\mathrm{HRP}$ 相比, $\mathrm{EB}_{9} / \mathrm{HRP}$ 复 合材料的吸附容量不是最高的, 但其反应速率常数 却是最大的, 达到 $0.0641 \mathrm{~min}^{-1}$, 约为 HRP 反应速 率常数的 2 倍。从这些数据来看, $\mathrm{EB} / \mathrm{HRP}$ 复合材料 的高降解活性主要来自于复合材料, 具有紧密接触 的界面, 这种界面作用使光生电子一空穴对快速分
离并传递到催化剂表面, 从而增强其光催化活性。

为了说明在光催化降解过程中 $\mathrm{RhB}$ 实现了染料 的无机矿化, 实验测试了催化剂 $\mathrm{EB}_{9} / \mathrm{HRP}$ 光降解 $\mathrm{RhB}$ 的紫外-可见吸收全谱。如图 3(c) 所示, RhB 染 料的最大吸收波长在 $554 \mathrm{~nm}\left(\lambda_{\max }\right)$ 处, 随着光照时 间的延长, $\mathrm{RhB}$ 的 $\lambda_{\text {max }}$ 发生了偏移、吸光度减弱且 未产生新的吸收峰, 这表明在降解过程中分别发生 了发色团的裂解和 N-去乙基化反应。除了对染料污 染物存在光降解行为外, 催化剂对非染料污染物也 存在很强的降解能力, 图 3(d) 为催化剂 HRP 和 $\mathrm{EB}_{9} / \mathrm{HRP}$ 降解 4-NP 的曲线图, 从图中可以看出纯 HRP 对 4-NP 有光降解能力, 而与膨润土复合后光 降解能力增强。由此可见, 所制备的复合催化剂不 仅对染料污染物分子存在很强的光降解能力, 而且 对非染料污染物也具有较强的光降解效果。

\section{4 催化剂机理分析}

膨润土是一种绝缘材料, 不能被可见光激发, 因而本身不具有光催化活性，催化剂性能的提高源 于 EB 对 HRP 的活化作用: 首先, 适量 EB 的添加改 进了 $\mathrm{EB}_{9} / \mathrm{HRP}$ 光催化剂对 $\mathrm{RhB}$ 分子的吸附性能, 增 加了复合催化剂的表面吸附, 为光催化反应提供了 更多的表面活性中心; 其次, EB 的静电相互作用可 以促进电子和空穴的分离，从而抑制电荷复合。 
为了分析光催化性能提高的机理, 利用电化学 工作站分析了 $\mathrm{HRP}$ 和 $\mathrm{EB}_{9} / \mathrm{HRP}$ 的光致 $i-t$ 曲线和电 化学阻抗谱(EIS)。通过瞬态光电流测量, 揭示了光 生电荷载流子在样品中的传输特性。图 4(a)显示了 纯 $\mathrm{HRP}$ 和 $\mathrm{EB}_{9} / \mathrm{HRP}$ 的瞬态光电流变化。纯 HRP 薄 膜光电极的感光电流密度约为 $0.75 \mu \mathrm{A} / \mathrm{cm}^{2}$ 。与 $\mathrm{EB}$ 复合后, HRP 的光响应得到明显改善, 光电流密度 显著提高，复合材料 $\mathrm{EB}_{9} / \mathrm{HRP}$ 的光电流密度达到 $1.5 \mu \mathrm{A} / \mathrm{cm}^{2}$, 是纯 HRP 的 2.0 倍, 说明负载 EB 后催 化剂的光生电子与空穴分离效率提高。

分别用 $\mathrm{HRP}$ 和 $\mathrm{EB}_{9} / \mathrm{HRP}$ 制备光电极, 并在光照 条件下以光电极为工作电极测试其电化学阻抗，考 察 $\mathrm{HRP}$ 和 $\mathrm{EB}_{9} / \mathrm{HRP}$ 催化剂的界面特性, 如电荷转移 的电阻大小和光生电子-空穴对的复合率。图 4(b) 给出了 $\mathrm{HRP}$ 和 $\mathrm{EB}_{9} / \mathrm{HRP}$ 的 EIS 能斯特图谱, 与纯 $\mathrm{HRP}$ 相比, $\mathrm{EB}_{9} / \mathrm{HRP}$ 的 EIS 图谱表现出更小的圆弧 半径, 表明光生电子-空穴对可以更好地分离, 并在 可见光照射下界面电荷转移更快。通常来说, 在 EIS 图中存在较小的圆弧半径, 表明电子转移电阻较 小、光生电子-空穴对的有效分离和光电极表面上界 面电荷转移更快。以上结果表明, 以 $\mathrm{EB}$ 为负载剂, 可以促进界面电荷的迁移和抑制光生电荷的复合, 有利于光催化。
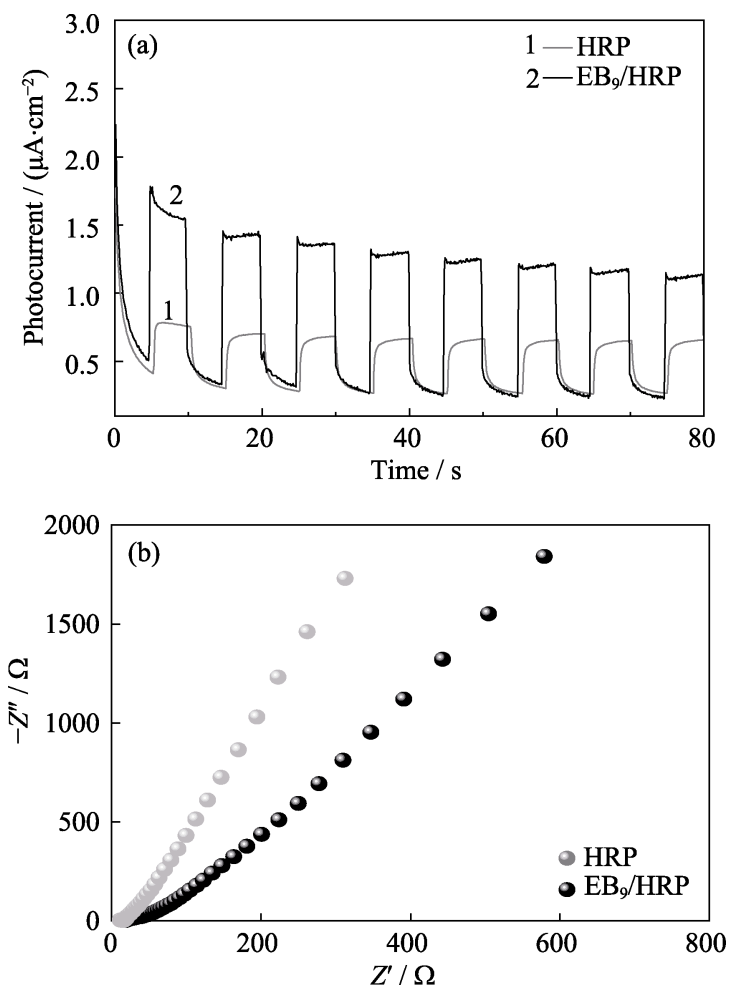

图 $4 \mathrm{HRP}$ 和 $\mathrm{EB}_{9} / \mathrm{HRP}$ 的瞬态光电流曲线(a)和电化学阻抗 图(b)

Fig. 4 (a) Photocurrent responses and (b) electrochemical impedance spectra of $\mathrm{HRP}$ and $\mathrm{EB}_{9} / \mathrm{HRP}$
为检测 $\mathrm{EB}_{9} / \mathrm{HRP}$ 样品在光催化反应中的活性成 分进行了自由基捕获实验, 实验中以苯醌(BQ)、叔 丁醇(TBA)和三乙醇胺(TEOA)分别为超氧自由基 $\left(\cdot \mathrm{O}_{2}{ }^{-}\right)$、羟基自由基 $(\cdot \mathrm{OH})$ 和光生空穴 $\left(\mathrm{h}^{+}\right)$的捕获剂。 从图 5(a)中可以看出, 加入苯醌后, $\mathrm{EB}_{9} / \mathrm{HRP}$ 对 $\mathrm{RhB}$ 的降解率从 $98.4 \%$ 降低到 $32.7 \%$, 而加入空穴捕获 剂三乙醇胺后, 对 $\mathrm{RhB}$ 的降解率直接降低到 $6.6 \%$, 说明在降解 $\mathrm{RhB}$ 实验过程中, $\mathrm{h}^{+}$是最主要的活性物 种, 而· $\mathrm{O}_{2}{ }^{-}$也是一种重要的中间活性物种, 加入叔 丁醇后降解率也有一定程度下降, 说明. $\mathrm{OH}$ 也是一 种中间活性物种。EB $9 / \mathrm{HRP}$ 光降解 RhB 的机理如下:

$$
\begin{gathered}
\mathrm{HRP}+h \mathrm{~h} \text { (可见光) } \rightarrow \mathrm{e}^{-}+\mathrm{h}^{+} \\
\mathrm{e}^{-}+\mathrm{O}_{2} \rightarrow \cdot \mathrm{O}_{2}^{-}
\end{gathered}
$$

$\mathrm{h}^{+}+$organic pollutants $\rightarrow$ degradation products

$\mathrm{O}_{2}^{-}+$organic pollutants $\rightarrow$ degradation products $(6)$

稳定性是评价催化剂的重要指标之一, 为确定 制备的光催化剂的稳定性, 取性能最好的 $\mathrm{EB}_{9} / \mathrm{HRP}$ 催化剂进行循环降解实验。如图 5(b)所示, 循环五 次后，催化剂对 RhB 的降解率仍然高达 96.8\%, 证 明 $\mathrm{EB}_{9} / \mathrm{HRP}$ 稳定性很好，是一种有实用前景的光催 化剂。
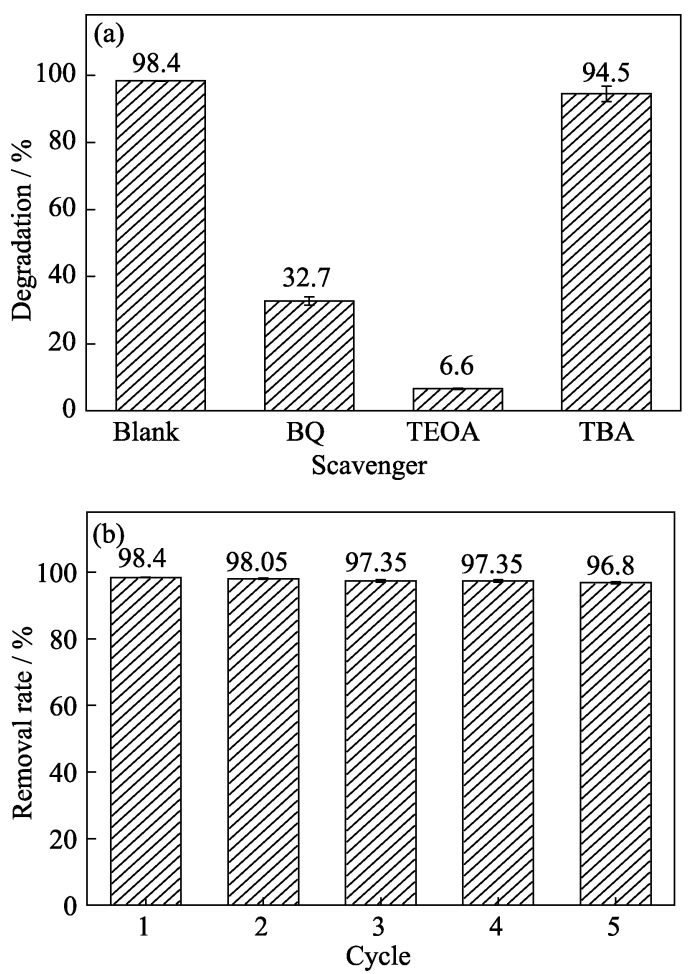

图 5 (a)不同捕获剂对 $\mathrm{EB}_{9} / \mathrm{HRP}$ 降解 $\mathrm{RhB}$ 的影响和(b) $\mathrm{EB}_{9} / \mathrm{HRP}$ 催化剂的循环实验图

Fig. 5 (a) Effect of different radical scavengers on the degradation efficiency of $\mathrm{RhB}$ for $\mathrm{EB}_{9} / \mathrm{HRP}$, and (b) cycling photocatalytic activity of $\mathrm{EB}_{9} / \mathrm{HRP}$

BQ: Benzoquinone; TBA: Tert-butyl alcohol; TEOA: Triethanolamine 


\section{3 结论}

通过简单水热法合成了 $\mathrm{EB} / \mathrm{HRP}$ 复合光催化 剂。该复合催化剂结构均匀, 具有更多的活性中心、 低的电阻以及更高的电荷转移能力。通过考察 $\mathrm{EB} / \mathrm{HRP}$ 复合光催化剂的吸附富集-定位光降解有 机污染物的能力, 发现该催化剂在可见光下对有机 污染物的吸附富集能力(吸附率达 $40 \%$ ) 和光催化活 性(降解速率为 $0.0641 \mathrm{~min}^{-1}$ ) 远高于 HRP(吸附率达 $20 \%$, 降解速率为 $0.0418 \mathrm{~min}^{-1}$ )。该结果表明, 将 $\mathrm{HRP}$ 负载在没有光响应能力的 $\mathrm{EB}$ 上显著增强了 HRP 的光催化性能, 为红磷光催化剂的改性提供了 一种新方法, 同时也为黏土作为催化剂载体的研究 提供了理论基础。

\section{参考文献:}

[1] WANG F, NG W K H, YU J C, et al. Red phosphorus: an elemental photocatalyst for hydrogen formation from water. Applied $\mathrm{Ca}$ talysis B: Environmental, 2012, 111: 409-414.

[2] WANG F, LI C, LI Y, et al. Hierarchical $\mathrm{P} / \mathrm{YPO}_{4}$ microsphere for photocatalytic hydrogen production from water under visible light irradiation. Applied Catalysis B: Environmental, 2012, 119-120: 267-272.

[3] YUAN Y P, CAO S W, LIAO Y S, et al. Red phosphor/g- $\mathrm{C}_{3} \mathrm{~N}_{4}$ heterojunction with enhanced photocatalytic activities for solar fuels production. Applied Catalysis B: Environmental, 2013, 140-141: 164-168.

[4] SHEN Z, SUN S, WANG W, et al. A black-red phosphorus heterostructure for efficient visible-light-driven photocatalysis. Journal of Materials Chemistry A, 2015, 3(7): 3285-3288.

[5] QI L, DONG K, ZENG T, et al. Three-dimensional red phosphorus: a promising photocatalyst with excellent adsorption and reduction performance. Catalysis Today, 2018, 314: 42-51.

[6] REN Z, LI D, XUE Q, et al. Facile fabrication nano-sized red phosphorus with enhanced photocatalytic activity by hydrothermal and ultrasonic method . Catalysis Today, 2020, 340(15): 115-120.

[7] ANSARI S A, ANSARI M S, CHO M H. Metal free earth abundant elemental red phosphorus: a new class of visible light photocatalyst and photoelectrode materials. Physical Chemistry Chemical Physics, 2016, 18(5): 3921-3928.

[8] SUN Y, REN Z, LIU Y, et al. Facile synthesis of ultrathin red phosphorus nanosheets with excellent photocatalytic performances. Materials Letters, 2019, 236: 542-546.

[9] SHI Z, DONG X, DANG H. Facile fabrication of novel red phosphorusCdS composite photocatalysts for $\mathrm{H}_{2}$ evolution under visible light irradiation. International Journal of Hydrogen Energy, 2016, 41(14): 5908-5915.

[10] BAI X, WAN J, JIA J, et al. Simultaneous photocatalytic removal of $\mathrm{Cr}(\mathrm{VI})$ and $\mathrm{RhB}$ over $2 \mathrm{D} \mathrm{MoS}_{2} /$ red phosphorus heterostructure under visible light irradiation. Materials Letters, 2018, 222: 187-191.

[11] BAI X, DU Y, HU X, et al. Synergy removal of Cr(VI) and organic pollutants over RP-MoS $/ \mathrm{rGO}$ photocatalyst. Applied Catalysis B: Environmental, 2018, 239: 204-213.

[12] WANG J, ZHANG D, DENG J, et al. Fabrication of phosphorus nanostructures $/ \mathrm{TiO}_{2}$ composite photocatalyst with enhancing photodegradation and hydrogen production from water under visible light. Journal of Colloid and Interface Science, 2018, 516: 215-223.

[13] WANG W, LI G, AN T, et al. Photocatalytic hydrogen evolution and bacterial inactivation utilizing sonochemical-synthesized g- $\mathrm{C}_{3} \mathrm{~N}_{4} /$ red phosphorus hybrid nanosheets as a wide-spectralresponsive photocatalyst: the role of type I band alignment. Applied Catalysis B: Environmental, 2018, 238: 126-135.

[14] FU J, XU Q, LOW J, et al. Ultrathin $2 \mathrm{D} / 2 \mathrm{D} \quad \mathrm{WO}_{3} / \mathrm{g}-\mathrm{C}_{3} \mathrm{~N}_{4}$ step-scheme $\mathrm{H}_{2}$-production photocatalyst. Applied Catalysis B: Environmental, 2019, 243: 556-565.

[15] CHAN D K L, YU J C, LI Y, et al. A metal-free composite photocatalyst of graphene quantum dots deposited on red phosphorus. Journal of Environmental Sciences (China), 2017, 60: 91-97.

[16] ZHU R, CHEN Q, ZHU R, et al. Sequestration of heavy metal cations on montmorillonite by thermal treatment. Applied Clay Science, 2015, 107: 90-97.

[17] RAMAKRISHNA KONDURU R, VIRARAGHAVAN T. Dye removal using low cost adsorbents. Water Science and Technology, 1997, 36(2/3): 189-196.

[18] EREN E, AFSIN B. An investigation of $\mathrm{Cu}(\mathrm{II})$ adsorption by raw and acid-activated bentonite: a combined potentiometric, thermodynamic, XRD, IR, DTA study. Journal of Hazardous Materials, 2008, 151(2/3): 682-691.

[19] ÖZCAN A S, ERDEM B, ÖZCAN A. Adsorption of Acid Blue 193 from aqueous solutions onto BTMA-bentonite. Colloids and Surfaces A: Physicochemical and Engineering Aspects, 2005, 266(1/2/3): 73-81.

[20] PERNYESZI T M, DéKáNY I. Photocatalytic degradation of hydrocarbons by bentonite and $\mathrm{TiO}_{2}$ in aqueous suspensions containing surfactants. Colloids and Surfaces A: Physicochemical and Engineering Aspects, 2003, 230(1/2/3): 191-199.

[21] MISHRA A, MEHTA A, KAINTH S, et al. Effect of different plasmonic metals on photocatalytic degradation of volatile organic compounds (VOCs) by bentonite/ $\mathrm{M}-\mathrm{TiO}_{2}$ nanocomposites under UV/visible light. Applied Clay Science, 2018, 153: 144-153.

[22] MESHRAM S, LIMAYE R, GHODKE S, et al. Continuous flow photocatalytic reactor using $\mathrm{ZnO}$-bentonite nanocomposite for degradation of phenol. Chemical Engineering Journal, 2011, 172(2/3): 1008-1015.

[23] PATIL S P, BETHI B, SONAWANE G H, et al. Efficient adsorption and photocatalytic degradation of Rhodamine $\mathrm{B}$ dye over $\mathrm{Bi}_{2} \mathrm{O}_{3}$-bentonite nanocomposites: a kinetic study. Journal of Industrial and Engineering Chemistry, 2016, 34: 356-363.

[24] MA J, HUANG D, ZHANG W, et al. Nanocomposite of exfoliated bentonite/g- $\mathrm{C}_{3} \mathrm{~N}_{4} / \mathrm{Ag}_{3} \mathrm{PO}_{4}$ for enhanced visible-light photocatalytic decomposition of Rhodamine B. Chemosphere, 2016, 162: 269-276.

[25] QU J G, LI N N, LIU B J, et al. Preparation of $\mathrm{BiVO}_{4} /$ bentonite catalysts and their photocatalytic properties under simulated solar irradiation. Materials Science in Semiconductor Processing, 2013, 16(1): 99-105.

[26] ABUKHADRA M R, SHABAN M, SAYED F, et al. Efficient photocatalytic removal of safarnin-O dye pollutants from water under sunlight using synthetic bentonite/polyaniline@ $\mathrm{Ni}_{2} \mathrm{O}_{3}$ photocatalyst of enhanced properties. Environmental Science and Pollution Research International, 2018, 25(33): 33264-33276.

[27] MA J, LIU Q, ZHU L, et al. Visible light photocatalytic activity enhancement of $\mathrm{Ag}_{3} \mathrm{PO}_{4}$ dispersed on exfoliated bentonite for degradation of rhodamine B. Applied Catalysis B: Environmental, 2016, 182: 26-32.

[28] HU Z, YUAN L, LIU Z, et al. An elemental phosphorus photocatalyst with a record high hydrogen evolution efficiency. Angewandte Chemie International Edition, 2016, 55(33): 9580-9585. 